\title{
Star-Like Branched Polyacrylamides by RAFT polymerization, Part II: Performance Evaluation in Enhanced Oil Recovery (EOR)
}

\author{
Benjamin Klemm, ${ }^{\dagger}$ Francesco Picchioni, ${ }^{\dagger, \ddagger}$ Patrizio Raffa, ${ }^{* \dagger}{ }^{\dagger}$ and Frank van Mastrigt ${ }^{\dagger, \ddagger}$ \\ ${ }^{\dagger}$ Department of Chemical Engineering, University of Groningen, Nijenborgh 4, 9747 AG Groningen, The Netherlands \\ ${ }^{\ddagger}$ Dutch Polymer Institute (DPI), P.O. Box 902, 5600 AX Eindhoven, The Netherlands
}

\section{Supporting Information}

ABSTRACT: In the present study the performance of a series of star-like branched polyacrylamides (SB-PAMs) has been investigated in oil recovery experiments to ultimately determine their suitability as novel thickening agent for enhanced oil recovery (EOR) applications. Hereby, SB-PAMs were compared with conventional linear PAM. The effect of a branched molecular architecture on rheology, and consequently on oil recovery was discussed. Rheological measurements identified unique properties for the SB-PAMs, as those showed higher robustness under shear and higher salt tolerance than their linear analogues. EOR performance was evaluated by simulating oil recovery in two-dimensional flow-cell measurements, showing that SB-PAMs perform approximately 3-5 times better than their linear analogues with similar molecular weight. The salinity did not influence the solution viscosity of the SB-PAM, contrarily to what happens for partially hydrolyzed polyacrylamide (HPAM). Therefore, SB-PAMs are more resilient under harsh reservoir conditions, which can make them attractive for EOR applications.

\section{INTRODUCTION}

Conventional oil extraction techniques, consisting of primary and secondary techniques recover at most $\sim 55 \%$ (typically between $20-40 \%{ }^{1}$ ) of the original oil in place (OOIP). ${ }^{2}$ In fact, an enormously large quantity $\left(7.0 \times 10^{12}\right.$ barrels $)$ of the OOIP remains embedded in mature oil fields. ${ }^{3}$ After exhaustion of conventional techniques, a tertiary method, known as enhanced oil recovery (EOR) is applied to target the remaining $\sim 45 \%$ of OOIP., ${ }^{2,45}$ EOR comprises a wide range of different methods ${ }^{6}$ specifically suited to extend reservoir lifetime by reducing the oil saturation in the reservoir below the residual oil saturation $\left(\mathrm{S}_{\mathrm{OR}}\right){ }^{4}$ Commonly, EOR can be classified in two main categories: thermal and nonthermal methods, ${ }^{4,7}$ whereas the latter, including chemical injection (e.g., polymers or surfactant solution), among others ${ }^{2,4}$ is most suitable for light oils $\left(\geq 20^{\circ}\right.$ American Petroleum Institute gravity index (API) $){ }^{4}$ In order to obtain a favorable mobility ratio, chemical EOR is based on increasing the viscosity of the displacement fluid (water). ${ }^{8}$ Hence, the smaller the difference in viscosity between the displacement fluid and the oil, the more efficient is the extraction of oil from the reservoir. ${ }^{7,9,10}$ However, to improve fluid viscosity, water-soluble polymers are applied as thickening agent to match the viscosity of the remaining oil. ${ }^{11-14}$ The most relevant water-soluble polymers in that respect are polyacrylamides (PAMs), whereas partially hydrolyzed polyacrylamide (HPAM) is by far the most commonly applied polymer in the EOR industry, ${ }^{3,15,16}$ due to its enormous viscosifying ability, which is attributed to its high molecular weight $\left(>1.0 \times 10^{6} \mathrm{Da}\right)$ and presence of charges. While high molecular weight HPAMs sufficiently increase the viscosity of injection water at low costs, ${ }^{9,17}$ several drawbacks arise in practicedue to the harsh conditions present in reservoirs. In fact, EOR polymer flooding is strongly influenced by mechanical (shear stress), chemical (salinity of the brine solution) and thermal (temperature) conditions, ${ }^{9,18}$ as well as the presence of divalent ions, ${ }^{18,19}$ injection times (at least 12 months) and geological properties of the reservoir. ${ }^{3}$ At high shear rates the viscosity of HPAM decreases rapidly, as the immobilized forces on the polymer result in a breakage of the polymeric backbone due to chain scission. ${ }^{20,21}$ Consequently, the higher the molecular weight (longer backbone) the more prone it is to degradation by mechanical forces. ${ }^{8}$ At increased salt concentrations, however, HPAM's viscosifying capability in aqueous solution drops significantly in consequence of reduced ionic repulsion, causing chain contraction. ${ }^{8,19,22}$ Furthermore, complexation of the carboxylate groups can result in polymer precipitation in the presence of divalent ions. ${ }^{8,23}$ In response, a lot of research has been performed in the last decades to modify rheological properties of HPAMs and hence complement their shortcomings, ${ }^{15,16,24,25}$ to ultimately find water-soluble polymers whose properties best fit EOR requirements. ${ }^{26}$ Specifically interesting in that respect are star, branched, and hyperbranched polymers, due to their unique rheological and physical properties in aqueous solutions compared with their linear analogues. ${ }^{27-31}$ Recently, we synthesized a series of water-soluble, star-like branched PAMs (SB-PAMs) by batch

Received: March 1, 2018

Revised: June 4, 2018

Accepted: June 8, 2018

Published: June 8, 2018 
copolymerization of acrylamide (AM) with $\mathrm{N}, \mathrm{N}^{\prime}$ methylenebis(acrylamide) (BisAM) as cross-linker or branching agent (BA), followed by chain extension with different levels of AM addition via reversible addition-fragmentation chain transfer (RAFT) polymerization. In brief, the macro RAFT agent ("core") was prepared by polymerizing AM together with BisAM as cross-linker in the presence of 3(((benzylthio)carbonothioyl)thio)propanoic acid (BCPA) as chain transfer agent (CTA), following the "core-first" approach. $^{32-34}$ The degree of branching was altered by increasing the ratio between monomer and comonomer, thus incorporating more BA to favor branching and to ultimately accomplish the transition to highly branched core structures. Hereafter, the macro RAFT agent was polymerized via chain extension with different levels of vinyl monomer (AM), to ultimately prepare the star-like PAM structures with a highly branched core moiety. The present work considers a follow-up study in which the performance of the recently synthesized SBPAMs in oil recovery are investigated, to ultimately determine their suitability as unconventional thickening agents for EOR applications. To render evidence to the former, SB-PAMs were set in direct comparison to conventional linear PAM. Hereby, it was emphasized to evaluate the effect of a branched molecular architecture on rheology and to correlate these properties to EOR performance.

\section{EXPERIMENTAL SECTION}

Chemicals. The crude oil used for the oil recovery experiments was heavy oil, which was kindly supplied by Shell Global Solution International B.V. The viscosity of the oil was $1,023 \mathrm{mPa}$.s at $20^{\circ} \mathrm{C}$, corresponding to API gravity below $22.3^{\circ}$, which has been adjusted to $110 \mathrm{mPa} . \mathrm{s}$ at $20{ }^{\circ} \mathrm{C}$ upon addition of cyclooctane. All other chemicals were reagent grade and used without further purification.

Synthesis of the Star-Like Branched Polyacrylamides. The synthesis of the star-like branched polymers is described in detail in our previous works. ${ }^{35}$ In brief, two polymerization steps were performed (1) the batch copolymerization of the macro RAFT agent (mCTA), which was conducted by charging a $25 \mathrm{~mL}$ round-bottomed flask with monomer (AM), vinyl monomer (BisAM), RAFT agent (BCPA) and initiator (4,4'- Azobis(4-cyanovaleric acid) (ACVA)), dissolved in a mixture of sodium acetate/acetic acid buffer solution ( $\mathrm{pH} \mathrm{5)}$ and ethanol (50:50 vol \%) and (2) the chain extension polymerization of the mCTA with AM to obtain SBPAMs, which was performed by charging a $250 \mathrm{~mL}$ threenecked flask with macro RAFT agent, monomer (AM) and initiator (ACVA), dissolved in sodium acetate/acetic acid buffer solution at $\mathrm{pH} 5$. Prior to initiation of the reaction, both systems (1) and (2) were deoxygenated by purging with nitrogen $\left(\mathrm{N}_{2}\right)$ gas for at least $30 \mathrm{~min}$ under vigorous stirring $(1,050 \mathrm{rpm})$ and subsequently placed in an (1) $70{ }^{\circ} \mathrm{C}$, (2) 80 ${ }^{\circ} \mathrm{C}$ oil bath and kept under nitrogen during the entire reaction time. The polymerization was terminated by exposure to ambient air and dilution with demineralised water. In case of (1) the product was isolated by evaporation at low pressure $(<100 \mathrm{mbar}$ ) and then redissolved. Afterward, the product (for both (1) and (2)) was precipitated by washing the polymer in an excess amount of methanol. Subsequently, the precipitated polymer was decanted and dried in an oven at $70{ }^{\circ} \mathrm{C}$. An overview of the results for the chain extension polymerization with different levels of AM is shown in Table 1. Polymers are referred to by their entry number as listed in Table 1 . Of
Table 1. Chain Extension Polymerization of Acrylamide with Macro RAFT Agent under Different Levels of AM Addition

$\begin{array}{cccll} & \text { mCTA }^{a}[\mathrm{BisAM}]_{0}: & \begin{array}{c}\text { extension } \\ {[\mathrm{AM}]_{0}}\end{array} & {[\mathrm{M}]_{\mathrm{n}, \text { theor. }}{ }^{b}} & {[\mathrm{M}]_{\mathrm{n}, \mathrm{GPC}}} \\ \text { entry } & 1: 99 & 5 & 11616 & 19120 \\ 1 & 1: 98 & 5 & 277442 & 352900 \\ 2 & 1: 49 & 5 & 338174 & 378500 \\ 3 & 1: 39 & 5 & 351198 & 460500 \\ 4 & 1: 29 & 5 & 401148 & 476000 \\ 5 & 1: 24 & 5 & 420263 & 504100 \\ 6 & 1: 19 & 5 & 458913 & 491200 \\ 7 & 1: 17 & 5 & 512149 & 544400 \\ 8 & 1: 14 & 5 & 552253 & 514100 \\ 9 & 1: 11 & 5 & 698364 & 573100 \\ 10 & 1: 9 & 5 & 727683 & 761000 \\ 11 & 1: 14 & 10 & 1127195 & 829000 \\ 12 & 1: 11 & 10 & 1387428 & 990900 \\ 13 & 1: 9 & 10 & 1317686 & 1003000 \\ 14 & 1: 7 & 10 & 2186704 & 1250000 \\ 15 & 1: 14 & 15 & 1954299 & 1243000 \\ 16 & 1: 11 & 15 & 1917810 & 1142000 \\ 17 & 1: 9 & 15 & 2111936 & 1072000 \\ 18 & 0 & 60 & 2365525 & 1143000 \\ 19^{c} & 0 & 10 & 3340000^{e} & 1110000 \\ 20^{d} & 0 & 5 & 5\end{array}$

${ }^{a}$ Parent macro RAFT agent (mCTA) ratio of $[\mathrm{BisAM}] /[\mathrm{AM}]$. ${ }^{b}$ Calculated from the linear equivalent theoretical molecular weight formula proposed by Stenzel et al. ${ }^{37}{ }^{c}$ Synthesized with a macro RAFT agent containing no BisAM. ${ }^{d}$ Radical polymerization without RAFT agent. ${ }^{e}$ Molecular weight estimation based on intrinsic viscosity measurements.

specific interest are entry 19 and 20. Entry 19 is a chain extension of a linear mCTA, offering a linear polymer. Entry 20 is polymerized under similar conditions as described for the SB-PAMs, however, in absence of a mCTA. Therefore, this polymer is obtained by a regular free radical polymerization. Based on intrinsic viscosity measurements $\left(K=4.9 \times 10^{-5}\right.$, a $=$ $0.80),{ }^{36}$ the $M_{\mathrm{w}}$ of the entry 20 was estimated to be 3340000 Da.

Rheological Characterization. Rheological measurements were performed on a HAAKE Mars III (ThermoScientific) rheometer, equipped with a cone-and-plate geometry (diameter $60 \mathrm{~mm}$, angle $2^{\circ}$ ) at $20{ }^{\circ} \mathrm{C}$. Aqueous polymer solutions were prepared by dissolving preweighed polymer in demineralised water at the desired weight percentage (typically $2.0 \mathrm{wt} \%$ ) and thereafter stirred until a homogeneous solution was obtained. Viscometric measurements, such as shear viscosity measurements (dependency of viscosity on shear rate) or viscoelasticity measurements were performed with shear rate variations of $0.1-1700 \mathrm{~s}^{-1}$ and frequency ranging from $6.37 \times 10^{-3}-15.92 \mathrm{~Hz}(0.04-100 \mathrm{rad} / \mathrm{s})$, respectively. In addition, prior to all viscoelasticity measurements an oscillation stress sweep was performed in order to determine the linear response region for each polymer frequency sweep. ${ }^{38}$ Hereafter, the storage modulus $\left(G^{\prime}\right)$ and loss modulus $\left(G^{\prime \prime}\right)$ measurements, that is, the complex dynamic viscosities (viscoelasticity) were measured according to the individual premeasured linear response region of the polymer solution (typical shear stress was $10 \mathrm{~Pa}$ ).

Flow-Cell Experiments. Dead end oil recovery simulations were performed using a 2-dimensional flow-cell, consisting of an aluminum bottom and a transparent plastic 


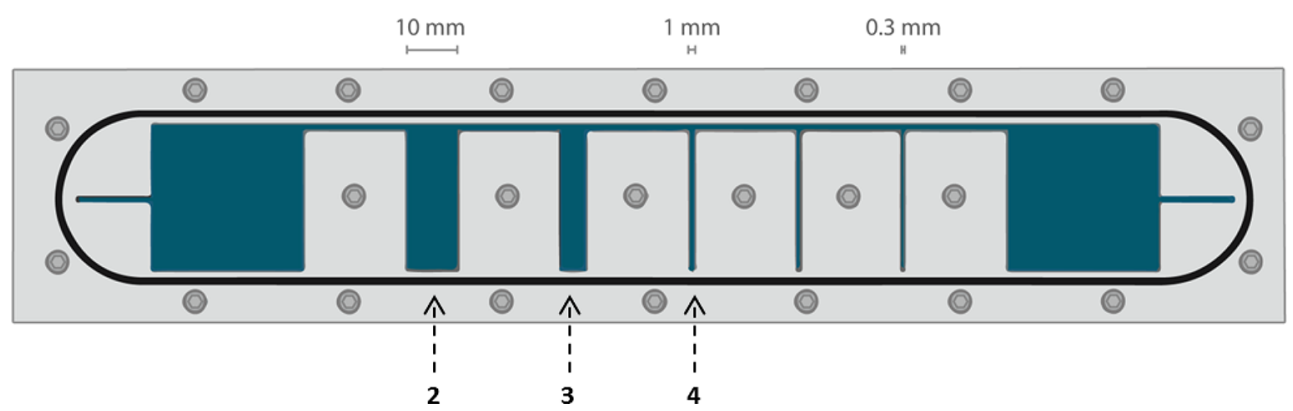

Figure 1. Schematic representation of the two-dimensional flow-cell (top view). Adapted with permission from ref. ${ }^{40}$ Copyright 2013 ACS.

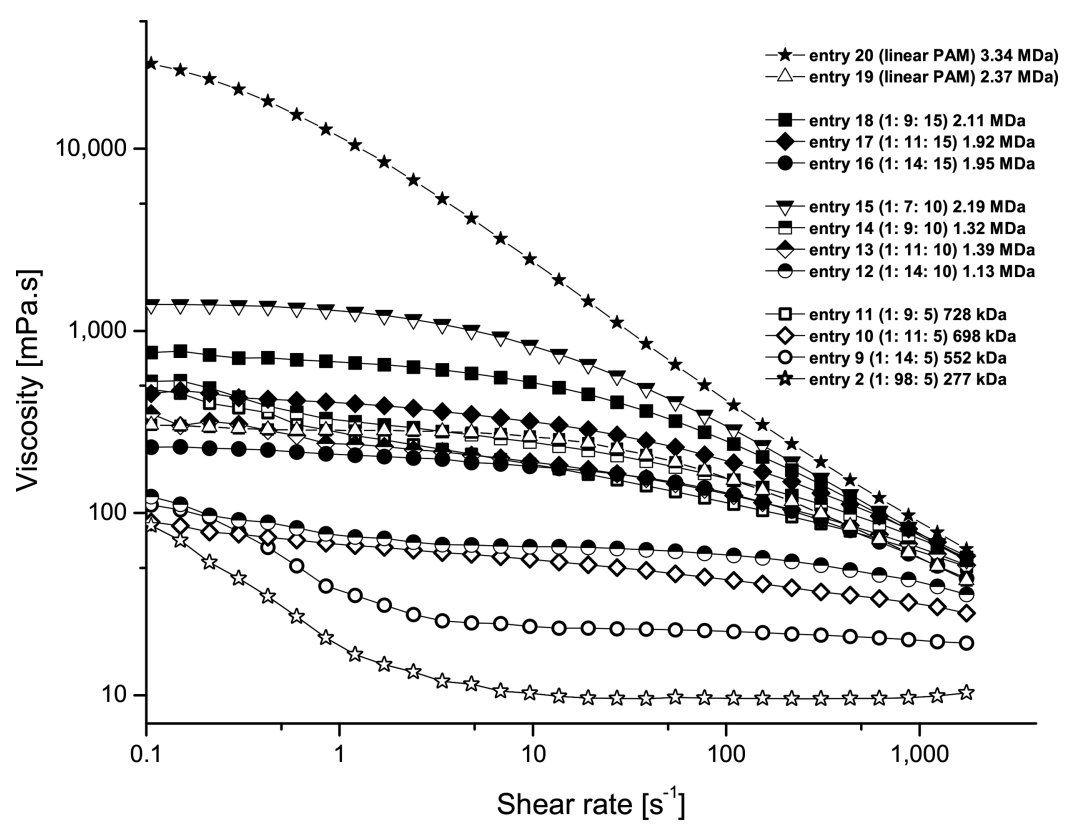

Figure 2. Viscosity as a function of shear rate for the differently prepared SB-PAMs (label X:Y:Z, corresponds to [BisAM]/[AM] ratio (X:Y) and (Z) to the addition of AM in grams) and linear PAMs (entry 19 (RAFT) and 20 (free radical polymerization)). Polymers were measured at a concentration of $2 \mathrm{wt} \%$.

top cover with size varied chambers to consecutively simulate dead end pores in oil reservoirs. The flow-cell setup (Figure 1) has been adapted from the original, presented by Niu et al. ${ }^{39}$ and was evaluated for EOR by Wever et al. ${ }^{40}$ The depth of the chambers (designated as dark blue in Figure 1) is set at 0.5 $\mathrm{mm}$. The experimental procedure was as follows: first, the flowcell was filled with crude oil. Here, it must be noted that the crude oil used for the experiment has been diluted with cyclooctane from a viscosity of $1,023 \mathrm{mPa} . \mathrm{s}$ to a viscosity of $110 \mathrm{mPa} . \mathrm{s}$ at $20{ }^{\circ} \mathrm{C}$, measured at constant shear rate of 9.63 $\mathrm{s}^{-1}$. Afterward, the flow-cell was flooded either by water (reference) or polymer solution using a pump to steadily inject the solution. The concentration of polymer was chosen as such, that the resulting polymer solution viscosity matched that of the crude oil. The flow rate was set to $1.00 \mathrm{~mL} /$ hour and experiments were continued for at least $24 \mathrm{~h}$ to reach steady state. Flow-cell measurements were graphically evaluated by taking high definition photographs from a set height point. The image analysis was performed using Adobe Photoshop CS6 via the 'pixel count' option to determine the amount of remaining crude oil in the chambers and consequently calculate the oil recovery according to

$$
\begin{aligned}
\text { oil rec\% } \% & \left(100-\frac{100 \cdot \text { pixel }_{\text {total }}}{\text { pixel }_{\text {remaining }}}\right)_{\text {polymer flood }} \\
& -\left(100-\frac{100 \cdot \text { pixel }_{\text {total }}}{\text { pixel }_{\text {remaining }}}\right)_{\text {water flood }}
\end{aligned}
$$

where the oil recovery (\%) is the percentage of oil recovered during polymer flood minus the water flood reference. It must be noted that for the calculation of the oil recovery only chamber 2, 3, and 4 were used as consecutive examples for dead-end pores (see Figure 1).

\section{RESULTS AND DISCUSSION}

Solution viscosity of the synthesized SB-PAM copolymers. The viscosity of the SB-PAM polymers dissolved in demineralised water as a function of shear rate was determined in the dilute/semidilute regime and is displayed in Figure 2. The degree of branching (incorporated amount of $\mathrm{BA}$ into the macromolecular core) and the concentration of AM used in the chain extension polymerization via RAFT, affected the apparent viscosity of the polymer in solution. As it can be observed, the flow curves for low molecular weight SBpolymers (see Table 1: e.g., entries 2 and 3 with a $M_{\mathrm{n}}$ of 
352900 and $378500 \mathrm{Da}$, respectively) exhibit shear thinning behavior at low shear rates, ${ }^{41-43}$ transforming to apparent Newtonian behavior at higher shear rates (at $\left.C<C^{* 44}\right){ }^{45}$ Upon shear the intramolecular associations are broken, leading to chain contraction and thus shear thinning. ${ }^{43,46,47}$

Similarly, for entry 20 (linear polymer synthesized by free radical polymerization with $[\mathrm{AM}] /[\mathrm{I}]=35,844 / 1$ at $80^{\circ} \mathrm{C}$ for $22 \mathrm{~min}$ ), the shear thinning effect can be very well observed. Although, entry 20 relates to the highest viscosity (due to chain entanglement), it represents the steepest drop in viscosity under increasing shear. The drop in viscosity at high shear rates illustrates that interchain associations dominate in the semidilute regime. ${ }^{48}$ The ability of disentanglement and overlapping of interchains overcomes the ability to reform aggregates at higher shear rates. ${ }^{24}$ Consequently, the effect of shear thinning is related to the disruption of the associating polymeric network, which results in isolation of polymeric structures. ${ }^{45}$ The latter is a well know effect and is frequently reported for pseudoplastic polymeric solutions. $^{42,49}$ In contrast, SB-polymers (e.g., entry 15 with a $M_{\mathrm{n}}$ of $\left.1250000 \mathrm{Da}\right)$, experience at low shear rates a weak shear thinning effect, which becomes more pronounced with increasing shear. It can be speculated that shear is insufficient to completely disrupt the chain extended densely branched network as the effect of shear thinning is rather weak. ${ }^{24}$ Rendering the latter toward the applicability of SB-polymers in the EOR industry, one will notice that although entry 20 exhibits the largest viscosity, it is instantly prone to shear thinning, while SB-polymers exhibit robustness under shear which is advantageous in the harsh conditions (high shear) of mature oil fields. On top of that, it has to be stated that the molecular weight of entry 20 is significantly higher than that of the SB-polymers. The molecular weight of entry 19 (linear PAM, chain extension of a linear mCTA) is in better comparison with some of the SB-PAMs. Even though the theoretical molecular weight of entry 19 is slightly higher than that of the branched equivalents, the viscosifying potential of the SB-PAMs is clearly illustrated as some of the SB-PAMs (entry 15,17 , and 18 ) offer a notably higher solution viscosity.

In Figure 3, the effect of branching and AM addition on the solution viscosity for the different SB-polymers is illustrated at a constant shear rate of $9.63 \mathrm{~s}^{-1}$, which is a relevant shear rate in EOR. ${ }^{39,50}$ As it can be observed, viscosity increases gradually with an increase of incorporated BA (BisAM) into the core structure at constant AM addition (entry 2-11 with 1:98:51:9:5, $\mathrm{X}: \mathrm{Y}: \mathrm{Z}$ corresponds to $[\mathrm{BisAM}] /[\mathrm{AM}]$ ratio $(\mathrm{X}: \mathrm{Y})$ and $(Z)$ to the chain extension polymerization addition of $A M$ in grams).

Note that the exceptions $(1: 19: 5$ and $1: 14: 5)$ can be rationalized by the lower observed monomer conversion $(84 \%$ and $75 \%$ ) compared with the other members of that generation (avg.: 87\%). In fact, more cross-linking in the core is related to the increase of local confinement of the polymer. In other words the polymer becomes stiffer (internal density increases), which in turn reduces the shear thinning effect and tends to constancy at the low shear spectra, ${ }^{51}$ while at high shear rates the intramolecular associations are further weakened, corresponding to the observed decrease in viscosity (Figure 2). Similar observations are made at AM amounts of 10 and $15 \mathrm{~g}$ for the same core moieties. Hence, the polymer with a higher degree of branching (1:9:5), thickens more efficiently (due to higher $M_{n}$ ) than SB-polymers with lesser associate branched structure $(1: 98: 5))^{52}$ More branching

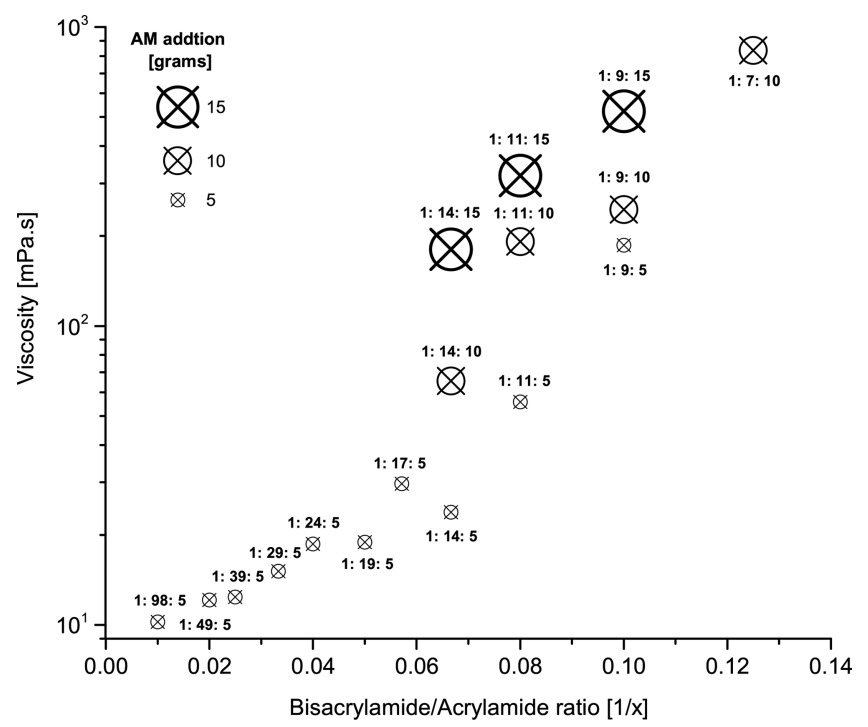

Figure 3. Viscosity (measured at $\dot{\gamma}=9.63 \mathrm{~s}^{-1}$ ) as a function of the $[\mathrm{BisAM}] /[\mathrm{AM}]$ ratio used to generate the core moieties and subsequent addition of $\mathrm{AM}$ in the chain extension polymerization to form the SB-polymers (label X:Y:Z, corresponds to [BisAM]/ $[\mathrm{AM}]$ ratio $(\mathrm{X}: \mathrm{Y})$ and $(\mathrm{Z})$ to the addition of $\mathrm{AM}$ in grams). Polymer concentration was 2 wt \% for all SB-polymer measurements.

points are available for extension with $\mathrm{AM}$, resulting into more polymeric arms around the core. The effect of AM addition with similar core structures (e.g., 1:9:5, 1:9:10, and 1:9:15), one can observe that higher amount of AM during chain extension results in enhanced thickening capability, which can be explained by the increase in $M_{n}{ }^{52}$ In that respect, polymeric chain extension contributes to pronounced intermolecular associations, thus the formation of a network structure of chains (large aggregates) with larger hydrodynamic volumes and ultimately higher solution viscosity. ${ }^{18}$ The higher hydrodynamic volume is caused by enhanced stretching of polymeric chains as a result of steric hindrance. ${ }^{53}$ These stretched network structures contribute significantly to the thickening behavior of the associative polymer (entry 18; 1:9:15). Those networks, however, are partially reversible at high shear rates (Figure 2), due to partial disruption of the intermolecular chains. ${ }^{51}$ The core density seems to be more relevant in regard to the thickening capability (entry 15; 1:7:10), which supports the theory that more branching points are available for extension, leading to more short polymeric arms and larger hydrodynamic volume ${ }^{53}$ and consequently higher viscosity.

Viscoelasticity. All SB-polymer samples were probed by oscillation experiments at polymer concentrations of $2 \mathrm{wt} \%$. The results of the measurement series are illustrated in Figure 4 and 5. In comparison to the viscoelastic behavior of the SBPAMs, linear PAMs (entry 19 and 20) were used as benchmark, whereby polymer samples were measured at equal concentration ( 2 wt \%). For entry 20, which was obtained by a free radical polymerization (Figure 4, A1), it can be observed that the storage $\left(G^{\prime}\right)$ and loss $\left(G^{\prime \prime}\right)$ moduli both increase with increasing oscillation frequency $(\omega)$, whereas the $G^{\prime \prime}$ approaches asymptotic behavior starting at $1 \mathrm{rad} / \mathrm{s}$ (e.g., in the terminal zone). The storage modulus $G^{\prime}$ is increasing at a faster rate than the loss modulus $G^{\prime \prime}$. At the intersection point of $G^{\prime}$ and $G^{\prime \prime}$, where the elastic modulus becomes more dominant than the viscous modulus the polymer behaves like a 


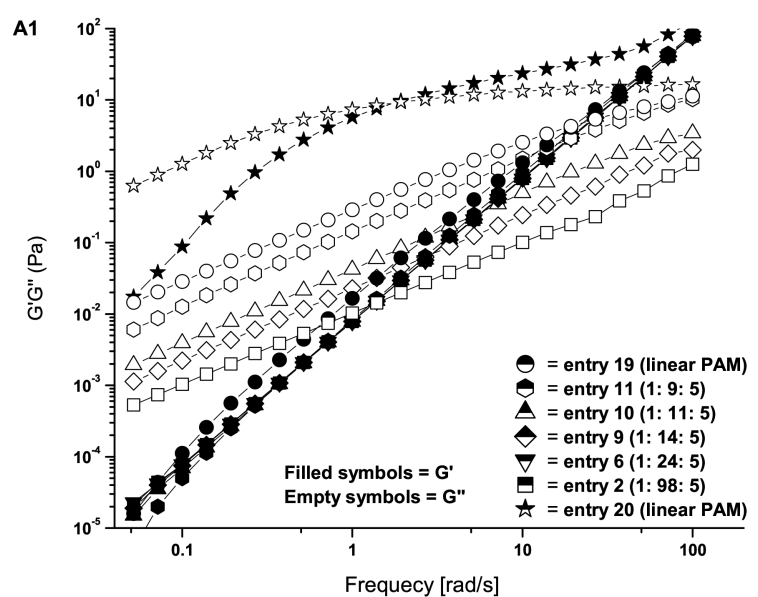

(a)

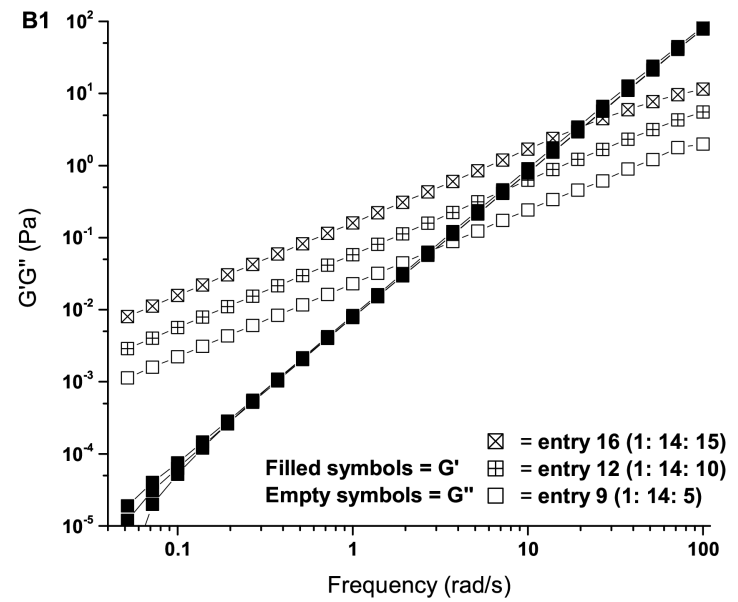

(c)

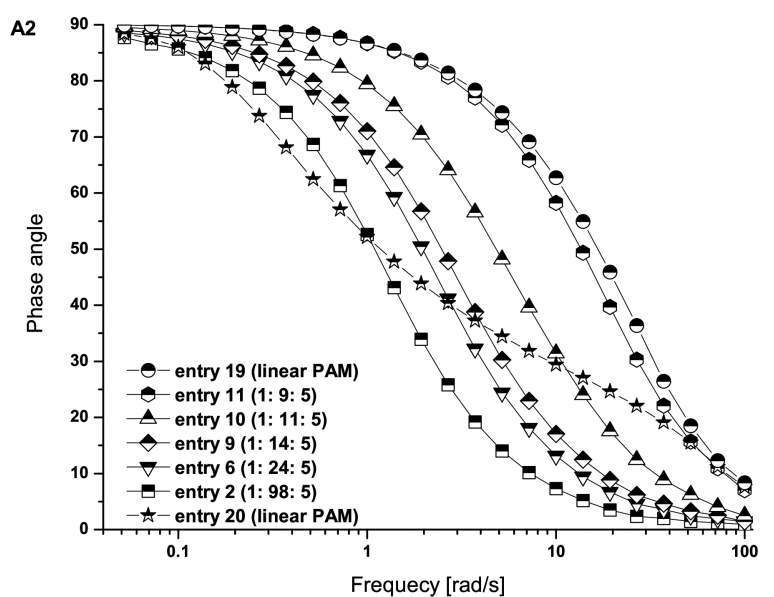

(b)

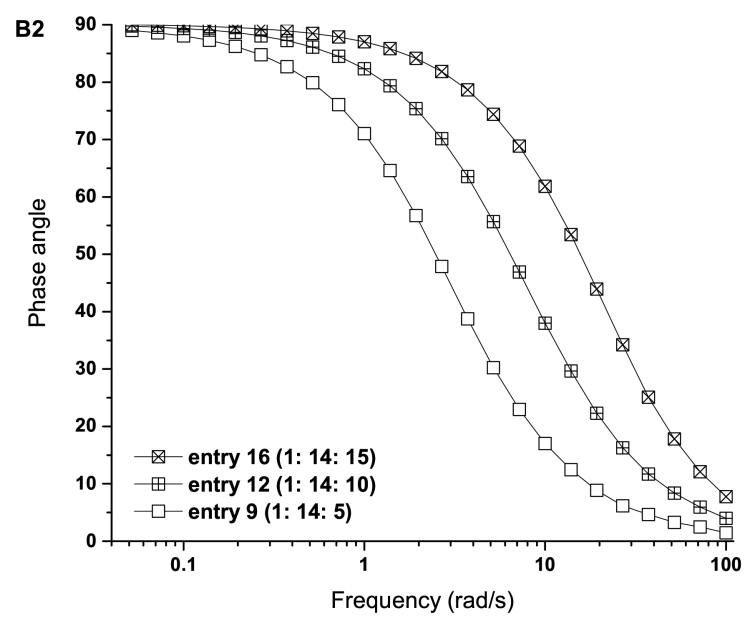

(d)

Figure 4. A1, $G^{\prime}$ and $G^{\prime \prime}$ of SB-PAMs with increased branching density at constant AM addition and linear PAM, A2 their respective phase angles. $\mathrm{B} 1, G^{\prime}$ and $G^{\prime \prime}$ of SB-PAMs with increased amount of AM addition at constant branching density (same core), B2 their respective phase angles.

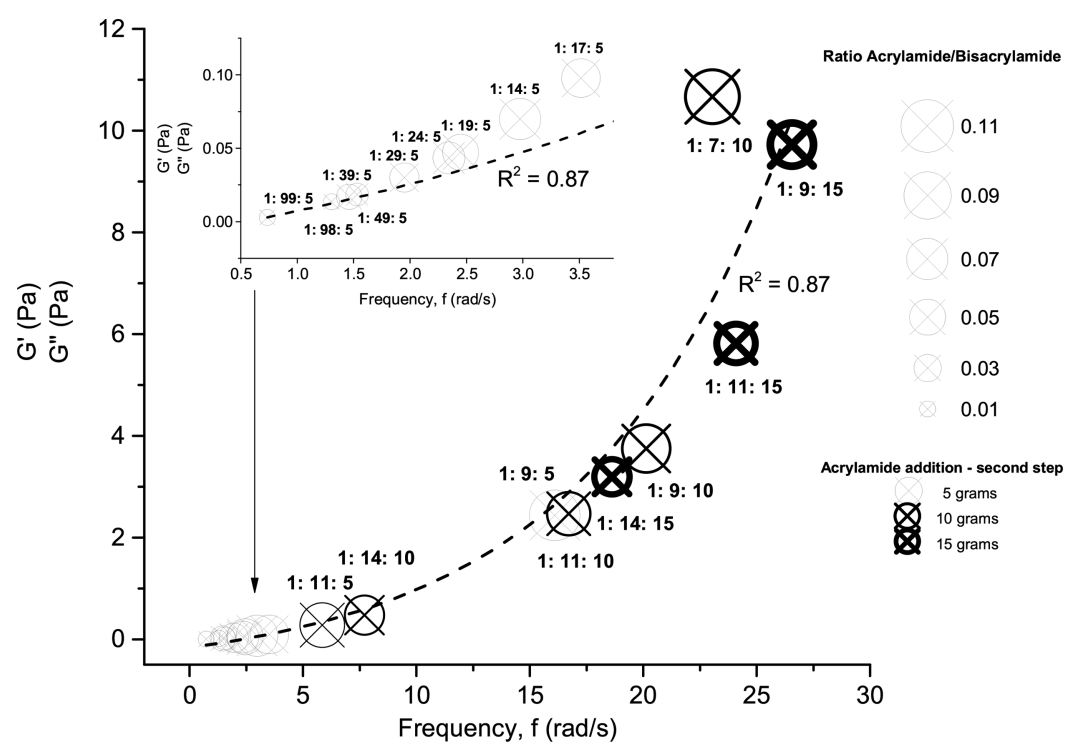

Figure 5. Viscoelastic intersection point of the differently prepared SB-PAMs with different branching density and different amounts of AM addition in the chain extension polymerization as a function of frequency and loss/storage modulus. 


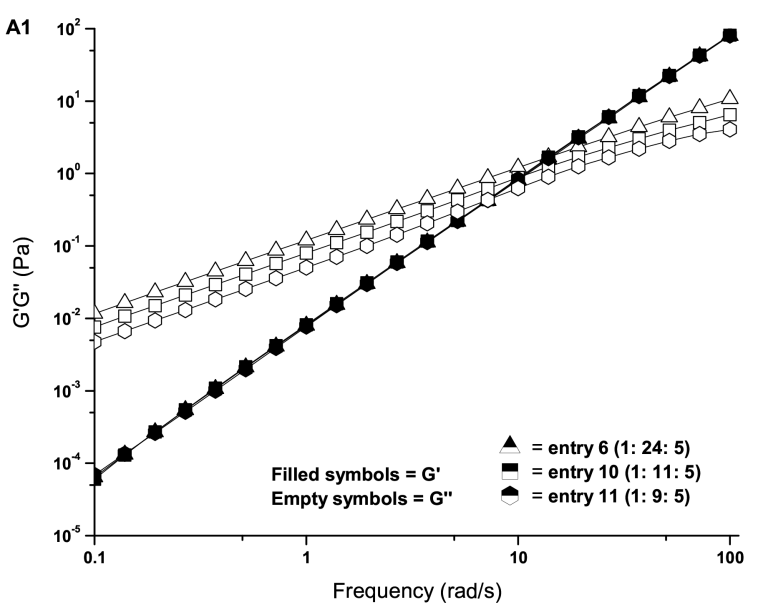

(a)

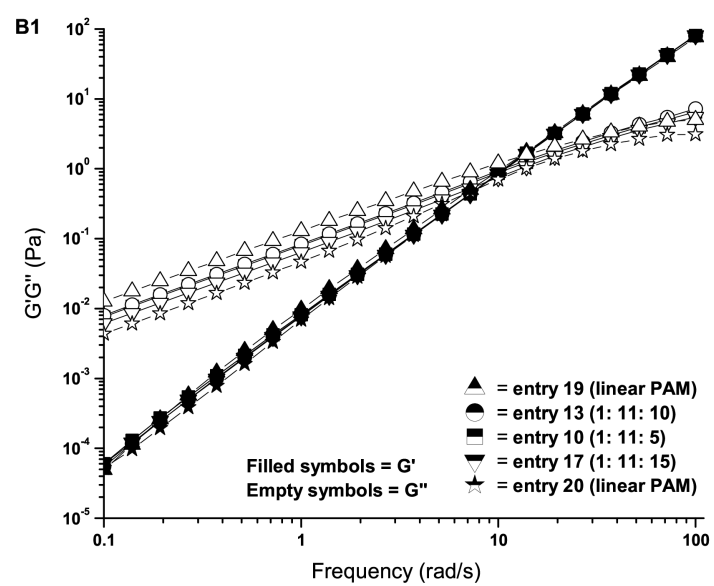

(c)

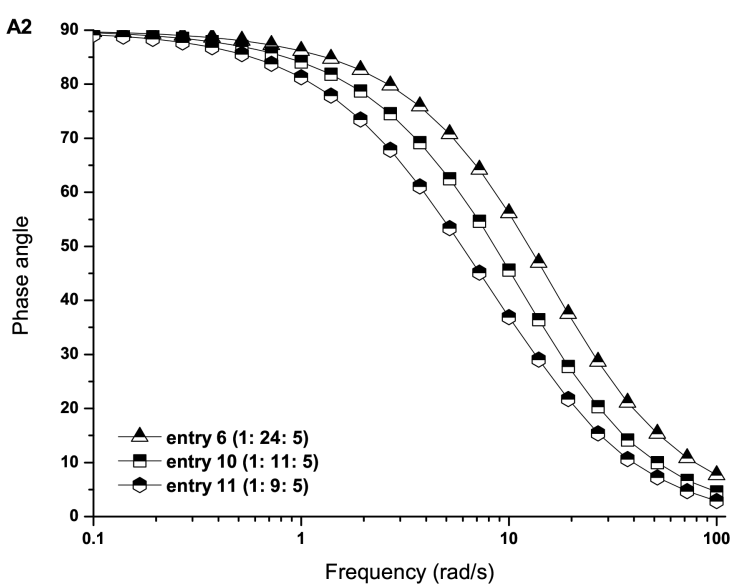

(b)

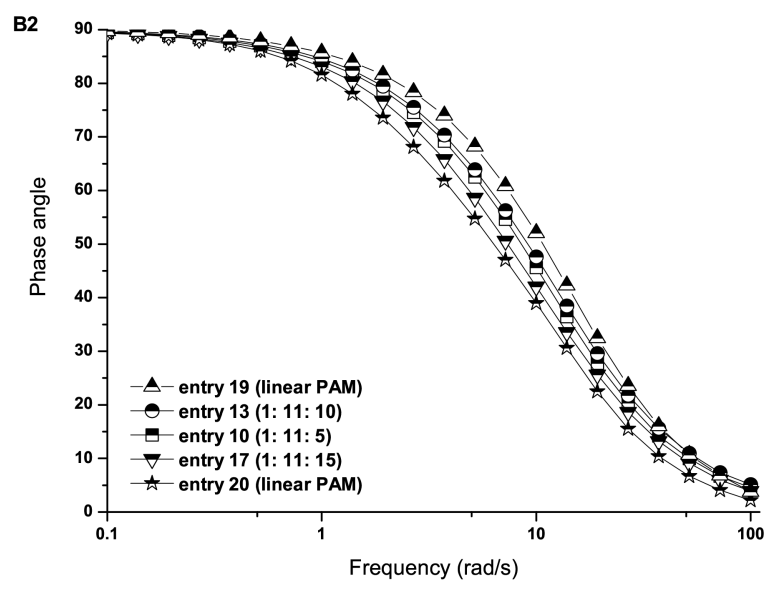

(d)

Figure 6. A1, $G^{\prime}$ and $G^{\prime \prime}$ of SB-PAMs with increased branching density at constant AM addition, A2 their respective phase angles (equal $\left.\eta_{0}\right)$. B1, $G^{\prime}$ and $G^{\prime \prime}$ of SB-PAMs with increased amount of AM addition at constant branching density (same core), B2 their respective phase angles (equal $\eta_{0}$ ).

viscoelastic gel. ${ }^{43,54}$ In contrast, the plots for SB-PAMs (entry $2,6,9,10$, and 11) at constant amount of AM addition and varying cross-link density (in the core structure), as well as linear entry 20, indicate that the magnitudes of $G^{\prime}$ and $G^{\prime \prime}$ increase at higher frequencies. The latter effect clearly illustrates the effect of the molecular weight on the rheological behavior, as linear entry 19 has a comparable molecular weight to the branched entries and was found to offer the least pronounced elastic behavior. Higher branching density in the core, results into an increase in the viscous modulus, while the elastic modulus remains constant. Significantly, all of the measured SB-polymers in Figure 4, A1, display viscoelastic fluid behavior throughout the complete frequency spectra with a $G^{\prime}$ that is directly proportional to $\omega^{2}($ slope $=2)$ and $G^{\prime \prime}$ which is proportional to the frequency $($ slope $=1)$. In fact, all SB-PAMs, convey this behavior at low frequencies (terminal response).

The comparison (Figure 4, A1) between different cross-link densities in the core at constant AM addition, suggests that more branching displays a more pronounced viscous response at equal concentration (higher phase angle, Figure 4, A2). Hence, higher frequencies are required in order for the elastic component to dominate the viscoelastic behavior for polymers with higher branching density in the core (Figure 5). The latter effect is supported by the low degree of elastic behavior as displayed by linear entry 19 (polymerized without BisAM in the mCTA). Similar observations are made for increased AM addition in the polymeric chain extension at constant core moieties (Figure 4, B1/B2).

However, the results can be disguised by the difference in viscosity. Therefore, Figure 6 shows the effect of branching density and AM addition at equal viscosity $\left(\eta_{0}\right)$ with different concentrations. In fact, from plot A1 and A2 in Figure 6, one will notice that higher branching density in the core corresponds to a more elastic behavior of the polymer solution at equal viscosity (Figure 6, A2). The latter finds agreement with earlier results, since higher cross-link density suggests that more branching points are available for extension, thus more/ short polymeric arms are formed, instead of longer polymeric arms and thus at low frequencies elasticity dominates $\left(G^{\prime}>\right.$ $\left.G^{\prime \prime}\right)$. The increase of the added amount of AM shows only minor effects on the viscoelasticity (Figure 6, B1) of the polymer and its corresponding phase angle (B2). However, the increase in the length of the polymeric arms (higher AM addition) leads to higher viscosity with lower concentration, causing a decrease in the loss modulus, which in turn shifts the 


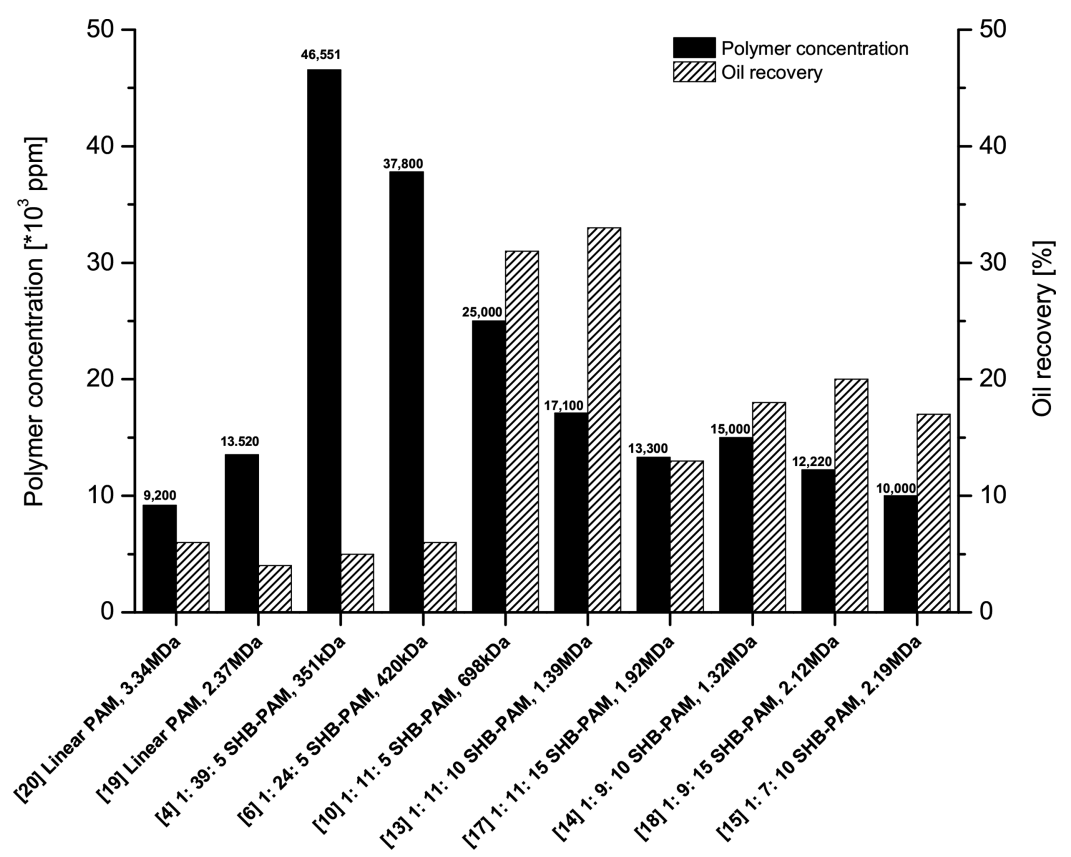

Figure 7. Additional oil recovery out of dead-end zones from the two-dimensional flow-cell after water flood.

phase angle to lower frequencies (polymer is more elastic). This phenomenon is in line with observations of polystyrenes in the melt. ${ }^{55}$ In fact, the terminal relaxation time increases with an increase of AM addition. ${ }^{53}$ On top of that, this effect is clearly illustrated by linear entries 19 and 20. As shown in Figure 6, B2, the linear PAM with a higher molecular weight (entry 20) offers a more pronounced elastic behavior, whereas linear entry 19 shows a relatively low degree of elasticity.

Flow-Cell Experiments. EOR performance of the synthesized SB-polymers was investigated by oil recovery simulation experiments using a two-dimensional flow-cell. Hereby, the polymer's capability to recover oil out of dead end zones was evaluated (see chamber 2, 3, and 4 in Figure 1) and subsequently calculated by eq 1 . The viscosity of the polymer solutions was matched to the viscosity of the crude oil (at 20 ${ }^{\circ} \mathrm{C}$ and a shear rate of $9.63 \mathrm{~s}^{-1}$ ) by altering the concentration. Different polymers were evaluated, in order to consequently determine the performance in relation to the architecture. SBpolymers were benchmarked against two linear polyacrylamides of comparable (entry 19) and higher (entry 20) molecular weight (as has been outlined earlier). The results of the simulations are summarized in Figure $7\left(M_{n}\right.$ is presented in Da for brevity), whereas the viscoelasticity measurements of the polymer solutions are displayed in Figure 8.

As can be observed, different oil recoveries are achieved with different polymer solutions. The polymer solutions, labeled 20 , 19,4 , and 6 , show the lowest improvement in oil recovery with $\sim 4-6 \%$. Although recovery is similar for those solutions, large differences exist in the used polymer concentrations. Specifically high concentrations were used for the "less branched" SB-polymers (low amounts of incorporated BA) with low AM addition in the chain extension polymerization to match the viscosity of the crude oil (entry 4 and 6 with a ratio of $[$ BisAM $] /[\mathrm{AM}]=1: 39,1: 24$ respectively). Obviously, those polymers also exhibit much lower molecular weights. However, the concentration of the entry 20 (linear PAM, with a $M_{\mathrm{w}}$ that is 8-10 times higher than that of entry 4 and 6) is much lower than that of the SB-polymers, while oil recovery is similar. The

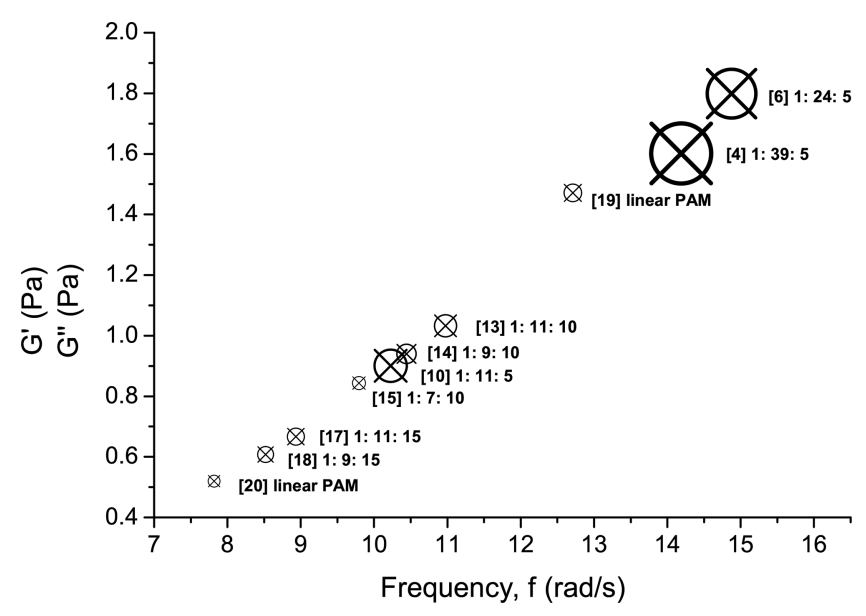

Figure 8. $G^{\prime}$ and $G^{\prime \prime}$ as a function of the frequency for the polymer solutions used in the flow-cell experiments. The labels $\mathrm{X}: \mathrm{Y}: \mathrm{Z}$, correspond to $[\mathrm{BisAM}]:[\mathrm{AM}]$ ratio for the copolymerization of the core structures $(\mathrm{X}: \mathrm{Y})$ respectively, while $(\mathrm{Z})$ corresponds to the level of AM added during the chain extension step to form the SB-polymers (X:Y:Z).

latter can be explained by the higher concentration at which the polymer coils start to overlap, generally at 100 up to 5000 ppm, ${ }^{56}$ which in turn positively affects the elastic response of the polymer ${ }^{57}$ and consequently enhances oil recoveries. The effectiveness (in terms of oil recovery) of the degree of branching can be better evaluated (based on [BisAM]/[AM] ratio) by comparing the oil recovery of entry 20 (linear) with the one for 1:7:10 SB-PAM (entry 15). The polymer is $\sim 3$ times more efficient in recovering oil than the linear one, at almost equal concentrations (and equal $\eta_{0}$ ). A comparison between entry 19 (linear PAM) and SB-PAM entries with a slightly lower molecular weight 15,17 , and 18 illustrates the advantages of the branched nature of the latter polymers. The linear polymer, despite requiring the highest polymer concentration, displays a significantly lower oil recovery of $4 \%$, versus $13-20 \%$ for the SB-PAMs. In context, from the 
viscoelasticity observations (Figure 8, intersection point for linear PAM at $9200 \mathrm{ppm}: 7.79,0.52$ ), entry 15 (intersection point at $10000 \mathrm{ppm}$ : $9.79,0.84$ ) is shown to be relatively elastic, which to some degree supports the general theory that elasticity benefits oil recovery. ${ }^{40}$ A clear effect of elasticity on oil recovery for the linear polymers, however, was not found. The oil recoveries of entry 19 and 20 are comparable, while there is a notable difference in their elasticity. SB-polymers with high concentrations of incorporated BA, demonstrated recoveries of $\sim 18-35 \%$ of the OOIP.

Despite the advanced recovery of SB-polymers, various differences in the performance can be observed from the obtained results. SB-PAMs with equal added amounts of AM in the extension step (e.g., entry 13,14, and 15), show that the degree of branching does not benefit the oil recovery after a ratio of $[\mathrm{BisAM}] /[\mathrm{AM}]=1: 11$ (entry 13). In fact, oil recoveries are decreasing, although more branching corresponds to higher molecular weights at equal AM additions (comparing entry 13,14, and 15). For AM addition (or in other words the effect of longer polymeric arms around the core) with the same branched core structures (e.g., entries 10, 13 , and 17 for $[\mathrm{BisAM}] /[\mathrm{AM}]=1: 11$ and entry 14 and 18 for $[\mathrm{BisAM}] /[\mathrm{AM}]=1: 9)$, the results suggest that longer polymeric arms slightly improve the oil recovery, but only upon a certain threshold. In particular, entry 10, 13, and 17 represent polymeric arms lengths, corresponding to 5, 10, and $15 \mathrm{~g}$ of $\mathrm{AM}$ addition, and are based on the same core structure ([BisAM $] /[\mathrm{AM}]=1: 11)$. The efficiency of 5 and $10 \mathrm{~g}$ of $\mathrm{AM}$ is twice as high as the one from $15 \mathrm{~g}$. In contrast, entry 15 and 18 , corresponding to 10 and $15 \mathrm{~g}$ of $\mathrm{AM}$ addition, show slight oil recovery improvements with increasing AM addition, as it was observed for the former one for 5 and $10 \mathrm{~g}$ (entry 10 and 13). Furthermore, the length of the polymeric arms seems to have an evenly important effect on the recovery. ${ }^{53}$ Wang et al. investigated the effect of elastic properties in EOR and concluded that besides normal stresses between oil and polymer, also shear stresses provoke interactions between the polymeric chains and the oil droplet, resulting in "pulling oil out" (pulling mechanism) of the dead end zones. ${ }^{58,59}$ The latter can be argued on the basis of the observed results. Specifically, interesting is the comparison between entry 10 and 13 , which correspond to the highest observed recoveries of $33 \%$ and $35 \%$, respectively. Although the theoretical molecular weight of the former is $50 \%$ lower and polymer concentration is $32 \%$ higher, the oil recoveries are similar. From these results, compared with the rest of SB-polymers and furthermore, looking closely to the effect of branching and AM addition, one might say that there is an optimum in improving the oil recovery for SB-PAM with an $[\mathrm{BisAM}] /[\mathrm{AM}]$ ratio $=1: 11$ core structure and AM addition of 5-10 g. Hereby, pronounced star-star coupling might positively influence the recovery performance resulting from the sweep effect of long chain branched structures (entry 10). At increased polymeric arm length (entry 13) this effect is enhanced due to stretching (entanglement) of the arms. ${ }^{53}$ In consequence of pronounced entanglement of the arms, more oil is recovered as the polymeric arms pull the oil out of dead ends (pulling effect). ${ }^{58,59}$

\section{CONCLUSIONS}

In the present study the performance of a series of star-like branched polyacrylamides (SB-PAMs) was investigated in oil recovery simulations. The rheological measurements of the SB-
PAMs showed unique properties compared with their linear analogues. Although a linear reference polyacrylamide (PAM) represented the highest solution viscosity, it is instantly prone to shear thinning, while in contrast SB-polymers exhibit robustness under shear which in turn is advantageous under the harsh conditions (high shear) during injection into oil fields. The observations from the viscoelasticity measurements, displayed that higher branching density in the core corresponds to more elastic behavior of the polymer at equal viscosity. The flow-cell experiments showed that SB-PAMs perform approximately 3-5 times more efficient (at comperable polymer concentration) than a linear analogue with a slightly higher molecular weight in recovering residual oil out of dead ends. Highest recoveries were $33-35 \%$ of the OOIP respectively. This effect is more relevant for larger arm length values due to stretching (entanglement) of the arms. In consequence of pronounced entanglement of the arms, more oil is recovered as the polymeric arms pull the oil out of dead ends (pulling effect). Finally, SB-PAM is more resilient to harsh reservoir conditions, which gives SB-PAMs a competitive advantage for EOR at the applied concentrations.

\section{ASSOCIATED CONTENT}

\section{S Supporting Information}

The Supporting Information is available free of charge on the ACS Publications website at DOI: 10.1021/acs.iecr.7b03368.

$\mathrm{G}^{\prime}, G^{\prime \prime}$, and phase angles of some of the SB-PAMs versus linear equivalents (PDF)

\section{AUTHOR INFORMATION}

\section{Corresponding Author}

*E-mail: p.raffa@rug.nl.

ORCID

Francesco Picchioni: 0000-0002-8232-2083

Patrizio Raffa: 0000-0003-0738-3393

\section{Notes}

The authors declare no competing financial interest.

\section{ACKNOWLEDGMENTS}

This research forms part of the research program of the Dutch Polymer Institute, Project 778.

\section{REFERENCES}

(1) Sandrea, I.; Sandrea, R. Global oil reserves-1: Recovery factors leave vast target for EOR technologies. Oil Gas J. 2007, 105, 44.

(2) Simon, R. Enhanced oil recovery: Definitions, fundamentals, applications, and research frontiers. Phys. Chem. Earth 1981, 13, 447460.

(3) Wever, D. A. Z.; Picchioni, F.; Broekhuis, A. A. Polymers for enhanced oil recovery: a paradigm for structure-property relationship in aqueous solution. Prog. Polym. Sci. 2011, 36, 1558-1628.

(4) Thomas, S. Enhanced oil recovery-an overview. Oil Gas Sci. Technol. 2008, 63, 9-19.

(5) Alvarado, V.; Manrique, E. Enhanced oil recovery: an update review. Energies 2010, 3, 1529-1575.

(6) Ayirala, S.; Yousef, A. A State-of-the-Art Review To Develop Injection-Water-Chemistry Requirement Guidelines for IOR/EOR Projects. SPE Prod. Oper. 2015, 30, 26-42.

(7) Lake, L. W. Enhanced Oil Recovery; Englewood Cliffs N.J, 1989.

(8) Taylor, K. C.; Nasr-El-Din, H. A. Water-soluble hydrophobically associating polymers for improved oil recovery: A literature review. J. Pet. Sci. Eng. 1998, 19, 265-280. 
(9) Choi, J.; Ka, D.; Chung, T.; Jung, J.; Koo, G.; Uhm, T.; Jung, S. H.; Park, S.; Jung, H. T. Evaluation of highly stable ultrahighmolecular-weight partially hydrolyzed polyacrylamide for enhanced oil recovery. Macromol. Res. 2015, 23, 518-524.

(10) Sheng, J. J. Transport of Chemicals and Fractional Flow Curve Analysis in Modern Chemical Enhanced Oil Recovery; Gulf Professional Publishing, 2011.

(11) Sheng, J.; Leonhardt, B.; Azri, N. Status of polymer-flooding technology. J. Can. Pet. Technol. 2015, 54, 116-126.

(12) Standnes, D.; Skjevrak, I. Literature review of implemented polymer field projects. J. Pet. Sci. Eng. 2014, 122, 761-775.

(13) Cardoso, O. R.; Lima Vidal, R. R.; de Lima, B. V.; de Carvalho Balaban, R. Polymer Flooding: The Influence of Polymer Chemical Structure and the Porous Medium. Macromol. Symp. 2016, 367, 3041.

(14) Saboorian-Jooybari, H.; Dejam, M.; Chen, Z. Heavy oil polymer flooding from laboratory core floods to pilot tests and field applications: Half-century studies. J. Pet. Sci. Eng. 2016, 142, 85-100.

(15) Shi, L.; Li, C.; Zhu, S. S.; Xu, J.; Sun, B. Z.; Ye, Z. B. Study on properties of branched hydrophobically modified polyacrylamide for polymer flooding. J. Chem. 2013, 2013. 1.

(16) Khune, G. D.; Donaruma, L. G.; Hatch, M. J.; Kilmer, N. H.; Shepitka, J. S.; Martin, F. D. Modified acrylamide polymers for enhanced oil recovery. J. Appl. Polym. Sci. 1985, 30, 875-885.

(17) Wei, B. Flow characteristics of three enhanced oil recovery polymers in porous media. J. Appl. Polym. Sci. 2015, 132.n/a

(18) Yahaya, G. O.; Ahdab, A. A.; Ali, S. A.; Abu-Sharkh, B. F.; Hamad, E. Z. Solution behavior of hydrophobically associating watersoluble block copolymers of acrylamide and N-benzylacrylamide. Polymer 2001, 42, 3363-3372.

(19) Levitt, D.; Pope, G. A. Selection and screening of polymers for enhanced-oil recovery. SPE Symp. Improved Oil Recovery. 2008. DOI: $10.2118 / 113845-M S$

(20) Seright, R. S.; Fan, T.; Wavrik, K.; Balaban, R. C. New insights into polymer rheology in porous media. SPE J. 2011, 16, 35-42.

(21) Brostow, W. Drag reduction and mechanical degradation in polymer solutions in flow. Polymer 1983, 24, 631-638.

(22) Cannella, W. J.; Huh, C.; Seright, R. S. Prediction of xanthan rheology in porous media. SPE Annu. Technol. Conf. Exhib. 1988. DOI: $10.2118 / 18089-\mathrm{MS}$

(23) Lai, N.; Dong, W.; Ye, Z.; Dong, J.; Qin, X.; Chen, W.; Chen, K. A water-soluble acrylamide hydrophobically associating polymer: Synthesis, characterization, and properties as EOR chemical. J. Appl. Polym. Sci. 2013, 129, 1888-1896.

(24) Yang, Q.; Song, C.; Chen, Q.; Zhang, P.; Wang, P. Synthesis and aqueous solution properties of hydrophobically modified anionic acrylamide copolymers. J. Polym. Sci., Part B: Polym. Phys. 2008, 46, $2465-2474$.

(25) Liu, X.; Jiang, W.; Gou, S.; Ye, Z.; Feng, M.; Lai, N.; Liang, L. Synthesis and evaluation of novel water-soluble copolymers based on acrylamide and modular $\beta$-cyclodextrin. Carbohydr. Polym. 2013, 96, $47-56$.

(26) Gou, S.; Yin, T.; Yan, L.; Guo, Q. Water-soluble complexes of hydrophobically modified polymer and surface active imidazoliumbased ionic liquids for enhancing oil recovery. Colloids Surf., A 2015, $471,45-53$.

(27) Voit, B. I.; Lederer, A. Hyperbranched and Highly Branched Polymer Architectures Synthetic Strategies and Major Characterization Aspects. Chem. Rev. 2009, 109, 5924-5973.

(28) Jesberger, M.; Barner, L.; Stenzel, M. H.; Malmström, E.; Davis, T. P.; Barner-Kowollik, C. Hyperbranched polymers as scaffolds for multifunctional reversible addition-fragmentation chain-transfer agents: A route to polystyrene-core-polyesters and polystyreneblock-poly (butyl acrylate)-core-polyesters. J. Polym. Sci., Part A: Polym. Chem. 2003, 41, 3847-3861.

(29) Jiménez-Regalado, E. J.; Cadenas-Pliego, G.; Pérez-Álvarez, M.; Hernández-Valdez, Y. Study of three different families of watersoluble copolymers: synthesis, characterization and viscoelastic behavior of semidilute solutions of polymers prepared by solution polymerization. Polymer 2004, 45, 1993-2000.

(30) Gao, C.; Yan, D. Hyperbranched polymers: from synthesis to applications. Prog. Polym. Sci. 2004, 29, 183-275.

(31) Tuncer, C.; Bütün, V. Highly cross-linked soluble star copolymers with well controlled molecular weights. Eur. Polym. J. 2015, 67, 292-303.

(32) Wang, D.; Wang, W. J.; Li, B. G.; Zhu, S. Semibatch RAFT polymerization for branched polyacrylamide production: Effect of divinyl monomer feeding policies. AIChE J. 2013, 59, 1322-1333.

(33) Gregory, A.; Stenzel, M. H. Complex polymer architectures via RAFT polymerization: From fundamental process to extending the scope using click chemistry and nature's building blocks. Prog. Polym. Sci. 2012, 37, 38-105.

(34) Chaffey-Millar, H.; Stenzel, M. H.; Davis, T. P.; Coote, M. L.; Barner-Kowollik, C. Design criteria for star polymer formation processes via living free radical polymerization. Macromolecules 2006, 39, 6406-6419.

(35) Klemm, B.; Picchioni, F.; Raffa, P.; Van Mastrigt, F. Star-Like Branched Polyacrylamides by RAFT polymerization - Part I: Synthesis and Characterization, 2018, submitted.

(36) Klein, J.; Conrad, K.-D. Molecular weight determination of poly (acrylamide) and poly (acrylamide-co-sodium acrylate). Makromol. Chem. 1978, 179, 1635-1638.

(37) Stenzel, M. H.; Davis, T. P. Star polymer synthesis using trithiocarbonate functional $\beta$-cyclodextrin cores (reversible additionfragmentation chain-transfer polymerization). J. Polym. Sci., Part A: Polym. Chem. 2002, 40, 4498-4512.

(38) Montalvo, G.; Valiente, M.; Rodenas, E. Rheological properties of the $\mathrm{L}$ phase and the hexagonal, lamellar, and cubic liquid crystals of the CTAB/benzyl alcohol/water system. Langmuir 1996, 12, 52025208.

(39) Niu, Y.; Jian, O.; Zhu, Z.; Wang, G.; Sun, G. Research on Hydrophobically Associating water-soluble polymer used for EOR. SPE Int. Symp. Oilfield Chem.. 2001. DOI: 10.2118/65378-MS

(40) Wever, D. A. Z.; Picchioni, F.; Broekhuis, A. A. Comblike polyacrylamides as flooding agent in enhanced oil recovery. Ind. Eng. Chem. Res. 2013, 52, 16352-16363.

(41) Hill, A.; Candau, F.; Selb, J. Properties of hydrophobically associating polyacrylamides: influence of the method of synthesis. Macromolecules 1993, 26, 4521-4532.

(42) Volpert, E.; Selb, J.; Candau, F. Influence of the hydrophobe structure on composition, microstructure, and rheology in associating polyacrylamides prepared by micellar copolymerization. Macromolecules 1996, 29, 1452-1463.

(43) Cram, S. L.; Brown, H. R.; Spinks, G. M.; Hourdet, D.; Creton, C. Hydrophobically modified dimethylacrylamide synthesis and rheological behavior. Macromolecules 2005, 38, 2981-2989.

(44) Galinsky, G.; Burchard, W. Starch fractions as examples for nonrandomly branched macromolecules. 2 . Behavior in the semidilute region. Macromolecules 1996, 29, 1498-1506.

(45) Tam, K. C.; Seng, W. P.; Jenkins, R. D.; Bassett, D. R. Rheological and microcalorimetric studies of a model alkali-soluble associative polymer (HASE) in nonionic surfactant solutions. J. Polym. Sci., Part B: Polym. Phys. 2000, 38, 2019-2032.

(46) Candau, F.; Selb, J. Hydrophobically-modified polyacrylamides prepared by micellar polymerization. Adv. Colloid Interface Sci. 1999, 79, 149-172.

(47) Corpart, J. M.; Candau, F. Aqueous solution properties of ampholytic copolymers prepared in microemulsions. Macromolecules 1993, 26, 1333-1343.

(48) Vittadello, S. T.; Biggs, S. Shear history effects in associative thickener solutions. Macromolecules 1998, 31, 7691-7697.

(49) McCormick, C. L.; Nonaka, T.; Johnson, C. B. Water-soluble copolymers: 27. Synthesis and aqueous solution behaviour of associative acrylamideN-alkylacrylamide copolymers. Polymer 1988, $29,731-739$. 
(50) Melo, D.; Aparecida, M.; Holleben, C. R.; Silva, I. G.; de Barros Correia, A.; Silva, G. A.; Rosa, A. J.; Lins, A. G. J.; Lima, D.; Carlos, J. Evaluation of Polymer-Injection Projects in Brazil, 2005.

(51) Omari, A.; Tabary, R.; Rousseau, D.; Calderon, F. L.; Monteil, J.; Chauveteau, G. Soft water-soluble microgel dispersions: Structure and rheology. J. Colloid Interface Sci. 2006, 302, 537-546.

(52) Zhang, Y. X.; Hwang, F. S.; Hogen-Esch, T. E. Macromolecular Complexes in Chemistry and Biology; Springer, 1994; pp 95-116.

(53) Wever, D. A. Z.; Polgar, L.; Stuart, M.; Picchioni, F.; Broekhuis, A. A. Polymer molecular architecture as a tool for controlling the rheological properties of aqueous polyacrylamide solutions for enhanced oil recovery. Ind. Eng. Chem. Res. 2013, 52, 16993-17005.

(54) Chen, H.; Tang, H.; Wu, X.; Liu, Y.; Bai, J.; Zhao, F. Synthesis, Characterization, and Property Evaluation of a Hydrophobically Modified Polyacrylamide as Enhanced Oil Recovery Chemical. J. Dispersion Sci. Technol. 2016, 37, 486-495.

(55) Gaylord, N. G.; Van Wazer, J. R. Viscoelastic Properties of Polymers; 1961.

(56) Stokes, R. J.; Evans, D. F. Fundamentals of Interfacial Engineering; John Wiley \& Sons, 1997.

(57) Guyot, A.; Chu, F.; Schneider, M.; Graillat, C.; McKenna, T. F. High solid content latexes. Prog. Polym. Sci. 2002, 27, 1573-1615.

(58) Wang, D.; Wang, G.; Wu, W.; Xia, H.; Yin, H. The Influence of Viscoelasticity on Displacement Efficiency-From Micro to Macro Scale. In SPE Annual Tech. Conf. Exhib., 2007.

(59) Wang, D.; Wang, G.; Xia, H. Large scale high visco-elastic fluid flooding in the field achieves high recoveries. In SPE Enhanced Oil Recovery Conference, 2011. 